\title{
Wettbewerbsverbote in ärztlichen Gesellschaftsverträgen - Anforderungen und Grenzen
}

\section{Einführung \\ $\nabla$}

Niedergelassene Ärzte können sich heute in vielfältigen Kooperationsformen, wie Berufsausübungsgemeinschaften und MVZ, zusammenschließen. Gesellschaftsrechtliche Zusammenschlüsse von freiberuflichen Ärzten müssen einerseits die berufs- und vertragsarztrechtlichen Vorgaben beachten. Daneben sind für die Ausgestaltung der Rechte und Pflichten der Gesellschafter die zivilrechtlichen Vorschriften der Gesellschaft bürgerlichen Rechts, insbesondere die $§ \S 703$ ff. BGB und die Bestimmungen des Partnerschaftsgesellschaftsgesetzes von Bedeutung.

Ein wesentlicher Aspekt der gesellschaftsvertraglichen Gestaltung sind die Folgen des Ausscheidens eines Arztes aus einer Gemeinschaftspraxis. Zur Absicherung der verbleibenden Ärzte wird im Gesellschaftsvertrag häufig ein Wettbewerbsverbot vereinbart, welches diese in zeitlicher, räumlicher und gegenständlicher Hinsicht vor einer konkurrierenden Tätigkeit des ausscheidenden Arztes schützen soll.

Diese im Vertrag zumeist als „Wettbewerbsklausel“ oder auch „Konkurrenzschutzklausel“ bezeichneten Regelungen untersagen dem ausscheidenden Gesellschafter die nachvertragliche Aufnahme seiner ärztlichen Tätigkeit in einem definierten Umfang und Zeitraum. Inhaltlich wird hierdurch dem ausscheidenden Gesellschafter, im Falle der Fortführung der Praxis durch die verbleibenden Gesellschafter, die Niederlassung während eines bestimmten Zeitraums in einem räumlich begrenzten Gebiet untersagt. Da die Grenzen und der Umfang solcher Verbote durch die Vertragspartner nur in be-

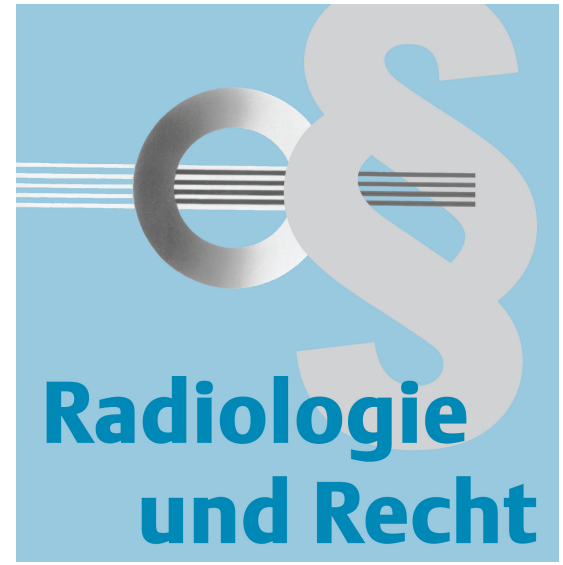

stimmten Grenzen individuell vereinbart werden können, sind nachvertragliche Wettbewerbsklauseln häufig Gegenstand gerichtlicher Entscheidungen.

In einer aktuellen Entscheidung hat sich das OLG Hamm (Beschl. v. 13.02.2012, Az.: I-8 W 16/12) mit der Rechtmäßigkeit eines Wettbewerbsverbotes im Gesellschaftsvertrag einer Gemeinschaftspraxis für Diagnostische Radiologie befasst. Die Entscheidung nehmen wir zum Anlass, einen Überblick über die unterschiedliche Rechtsprechung hinsichtlich der Wirksamkeit von vertraglich vereinbarten nachträglichen Wettbewerbsverboten zu 
geben. Zudem sollen neben den gesellschaftsrechtlichen Fragestellungen auch die arbeitsrechtlichen Aspekte von nachvertraglichen Wettbewerbsverboten dargelegt werden.

\section{Entscheidung des OLG Hamm}

Dem Beschluss des OLG Hamm lag der Antrag auf Erlass einer einstweiligen Verfügung eines Facharztes für diagnostische Radiologie gegen den früheren Mitgesellschafter einer radiologischen Gemeinschaftspraxis zugrunde, welche im Jahr 2004 in Form einer Gesellschaft bürgerlichen Rechts (GbR) gegründet worden war. Im Gesellschaftsvertrag wurde seinerzeit ein Wettbewerbsverbot mit folgendem Inhalt vereinbart:

„Dem ausgeschiedenen Gesellschafter ist es untersagt, sich innerhalb von 5 Jahren nach seinem Ausscheiden aus der Gesellschaft in X oder im Umkreis von X, als Arzt niederzulassen; gelegentliche Praxisvertretungen werden durch das Verbot nicht berührt.“

Ende des Jahres 2011 schied der Radiologe aus der Gesellschaft aus und ließ sich zum 01.01.2012, nur 4 Kilometer von der ehemaligen Gemeinschaftspraxis entfernt, als Facharzt für diagnostische Radiologie nieder, wogegen der in der Gesellschaft verbleibende Gesellschafter im Wege des Eilrechtsschutzes gerichtlich vorging.

\section{Zulässigkeitsvoraussetzungen von Wettbewerbsklauseln}

Nachvertragliche Wettbewerbsverbote sind zivilrechtlich insbesondere auf ihre Vereinbarkeit mit §138 BGB zu überprüfen, wonach ein Rechtsgeschäft, das gegen die guten Sitten verstößt, nichtig ist.

Nach der ständigen Rechtsprechung des BGH wird die Beurteilung der Sittenwidrigkeit von nachvertraglichen Wettbewerbsklauseln gemäß $\S 138$ Abs. 1 BGB durch das Grundrecht der Berufsfreiheit nach Art. 12 Abs. 1 GG mitgeprägt, welches auch die Berufsausübungsfreiheit des Einzelnen schützt. Danach sind nachvertragliche Wettbewerbsverbote nur dann wirksam, wenn sie durch ein schutzwürdiges Interesse des Berechtigten gefordert werden und hinsichtlich ihres räumlichen, zeitlichen und gegenständlichen Umfangs nicht das notwenige Maß überschreiten, mithin angemessen sind (vgl. BGH, Urt. v. 29.03.2003, Az.: II ZR 59/02; BGH, Urt. v. 08.05.2000, Az.: II ZR 308/98).

Die Rechtfertigung nachvertraglicher Wettbewerbsklauseln liegt allein im Schutz der Partner des ausgeschiedenen Gesellschafters vor einer illoyalen Verwertung der Erfolge der gemeinsamen Arbeit oder in dem Schutz vor einem Missbrauch der Ausübung der Berufsfreiheit. Schutzzweck ist daher gerade nicht, den ehemaligen Partner als potenziellen Konkurrenten auszuschalten (BGH, a.a.O.).

In diesem Zusammenhang ist zu beachten, dass das Anliegen der verbleibenden Gesellschafter, vor illoyalem Wettbewerb geschützt zu sein, nicht tangiert ist, soweit sich der ausscheidende Gesellschafter in hinreichender räumlicher Entfernung niederlässt und seinen Beruf ausübt. Dies gilt auch für den Fall, dass der ehemalige Gesellschafter auf einem nicht von der Gesellschaft ausgeübten Berufsfeld tätig wird (OLG Saarbrücken, Beschl. v. 17. 8. 2011, Az.: 1 U 184/11-52).

\section{Zeitliche Grenzen von Wettbe- werbsklauseln $\nabla$}

Die nachvertragliche Wettbewerbsklausel spricht in der Regel einen zeitlichen Geltungsbereich aus, welcher nach ständiger Rechtsprechung des BGH den Zeitraum von 2 Jahren nicht überschreiten darf. Begründet wird dieser begrenzte Zeitrahmen mit der Erwägung, dass der ausgeschiedene Gesellschafter nach Ablauf der 2 Jahre nicht mehr über besondere Kenntnisse verfügt, die er zum Nachteil der Gesellschaft nutzen könnte. Zudem wird davon ausgegangen, dass sich innerhalb des 2-jährigen Zeitraums die geknüpften Verbindungen zur ehemaligen Gesellschaft weitestgehend gelöst haben, sodass der ausgeschiedene Gesellschafter wie jeder andere Wettbewerber zu behandeln ist (BGH, Urt. v. 26.03.1984, Az: II ZR 229/83; Urt. v. 16.10.1989, Az.: II ZR 2/89; Urt. v. 29.10.1990, Az.: II ZR 241/89; Urt. v. 29.01.1996, Az.: II ZR 286/94).

Sollten die Vertragsparteien dennoch eine unangemessen lange Frist, welche 2 Jahre übersteigt, gewählt haben, führt dies regelmäßig nicht unmittelbar dazu, dass die Wettbewerbsklausel insgesamt gemäß $\S 138$ Abs. 1 BGB nichtig ist. Vielmehr wird die Klausel von den Gerichten im Wege der sog. „geltungserhaltenden Reduktion“ auf ein angemessenes Maß reduziert und bleibt somit wirksam, sofern sie im Übrigen rechtlich zulässig ist (BGH Urt. v. 14.07.1997, Az.: II ZR 238/96, NJW 1997, 3089; BGH Urt. v. 29.10.1990, Az.: II ZR 241/89, NJW 1991, 699).

Entsprechend den oben genannten Voraussetzungen stellt das OLG Hamm in seiner Entscheidung fest, dass der zeitliche Rahmen der Schutzfrist in der Regel nicht 2, maximal 3 Jahre, überschreiten darf. Die Verbotsdauer in der vorliegenden Klausel, sei daher mit 5 Jahren erheblich überschritten und damit als nicht mehr zulässig anzusehen.

\section{Räumliche Grenzen des Wettbe- werbsverbots $\nabla$}

Die Rechtsprechung zu den zulässigen räumlichen Grenzen nachvertraglicher Wettbewerbsklauseln ist dagegen uneinheitlich und richtet sich nach den Umständen des Einzelfalls. Für die Bestimmung eines zulässigen Umkreises der Wettbewerbsbeschränkung sind sowohl die Fachrichtung und die Größe der Gemeinschaftspraxis, als auch deren geografische Lage von Bedeutung. Das Wettbewerbsverbot darf prinzipiell nicht über den Einzugsbereich der bisherigen gemeinsamen Praxis hinausgehen.

Hinsichtlich der geografischen Lage ist das räumliche Ausmaß des Verbots abhängig davon, ob sich die Praxis im ländlichen Bereich oder in einem dicht besiedelten Stadtgebiet befindet. Die Einordnung eines Gebiets als dicht besiedelt oder als ländlich wird von den Gerichten unterschiedlich vorgenommen und ist vom Einzelfall abhängig. Das OLG Koblenz (Urt. v. 22.02.2012, Az.: 5 U 1233/11) hat zum Beispiel hinsichtlich einer nachvertraglichen Wettbewerbsklausel in einem Übernahmevertrag einer Zahnarztpraxis entschieden, dass ein räumliches Verbot im Umkreis von 9 Kilometern unbedenklich ist. Dagegen hat das LG Krefeld (Urt. v. 04.01.2007, Az.: 3 0 443/06) in Bezug auf das Ausscheiden einer Gesellschafterin aus einer orthopädischen Praxis ein räumliches Verbot in einem Radius von $10 \mathrm{~km}$ mit der Begründung als unzulässig angesehen, dass die Stadt mit ca. 220000 Einwohnern als dicht besiedelt anzusehen ist. 
Weiterhin ist die ausgeübte Fachrichtung der Gemeinschaftspraxis ein weiterer wichtiger Faktor für die Bemessung von zulässigen räumlichen Verbotsgrenzen. Der BGH hat im Fall einer Gemeinschaftspraxis mit 2 Fachärzten für Innere Medizin, Nephrologie ein Wettbewerbsverbot auf $2 \mathrm{~km}$ Luftlinie sowohl hinsichtlich der Tätigkeit der ausscheidenden Gesellschafterin als Internistin, als auch ein Verbot in Umkreis von $20 \mathrm{~km}$ bezüglich der Erbringung von Dialyseleistungen, als zulässig erachtet. Zur Begründung wird ausgeführt, dass die Durchführung von Dialysebehandlungen mit erheblichen finanziellen Aufwendungen verbunden sind und diese wirtschaftlich nur zu erbringen sind, wenn eine entsprechende Zahl von Patienten sie in Anspruch nimmt. Dies erfordere die Festlegung eines entsprechend großen Einzugsgebiets, welches mit $20 \mathrm{~km}$ nicht unangemessen groß sei (BGH, Urt. v. 07. 5. 2007, Az.: II ZR 281/05).

Das im Fall des OLG Hamm vereinbarte räumliche Verbot von $30 \mathrm{~km}$ bezog sich nicht auf den Umkreis ausgehend von der ehemaligen Praxis, sondern ausgehend vom gesamten Stadtgebiet. In diesem $\mathrm{Zu}-$ sammenhang führte das OLG Hamm aus, dass hierdurch dem ausgeschiedenen Radiologen ein nicht mehr verhältnismäßiger Ortsbereich, auch unter Einschluss von Gebieten anderer größerer Städte in der Umgebung, zur Ausübung seiner Tätigkeit verbleiben würde. Dies sei selbst unter Berücksichtigung des Einzugsbereiches der Gemeinschaftspraxis in der betroffenen Stadt nicht mehr gerechtfertigt.

Zusammenfassend lässt sich festhalten, dass bei der Aufnahme von räumlichen Grenzen in Wettbewerbsklauseln eine restriktive Festlegung der Entfernungen geboten ist, welche unter Abwägung beiderseitiger Interessen verhältnismäßig sein muss. Die äußerste Grenze dürfte in diesem Zusammenhang maximal der Einzugsbereich der ehemaligen Praxis sein. Darüber hinausgehende Entfernungen führen demgegenüber zur Unzulässigkeit der räumlichen Regelung.

$\mathrm{Zu}$ beachten ist außerdem, dass die Vornahme einer geltungserhaltenden Reduktion durch das Gericht auf ein zulässiges Maß bei infrage stehenden räumlichen Grenzen in Wettbewerbsklauseln nicht zulässig ist. Im Gegensatz zu einer unzulässigen Überschreitung der zeitlichen Grenzen, führt daher eine unzulässige räumliche Eingrenzung zu einer Nichtigkeit der gesamten Klausel gemäß $§ 138$ Abs. 1 BGB (BGH, Urt. v. 14.07.1997, Az.: ZR 238/96). Der ausscheidende Gesellschafter unterliegt in einem solchen Fall keinerlei Wettbewerbsverbot, sodass bei der Vereinbarung von räumlichen Grenzen Vorsicht geboten ist.

\section{Gegenständliche Grenzen eines Wettbewerbsverbots $\nabla$}

Die gegenständlichen Grenzen einer Wettbewerbsklausel werden insbesondere dann überschritten, wenn dem ausscheidenden Gesellschafter nicht nur die Tätigkeit in dem Fachgebiet untersagt wird, in dem er tätig ist, sondern auch seine Tätigkeit als niedergelassener Arzt überhaupt untersagt wird, da dies im Ergebnis zu einem Berufsverbot zu Lasten des ausscheidenden Gesellschafters führen würde (OLG Düsseldorf, Urt. v. 19.03.2007, Az.: I 9 U 46/07). Ein Verbot, welches sich auf die in der ehemaligen $\mathrm{Ge}$ meinschaftspraxis ausgeübte Facharzttätigkeit beschränkt und sich somit am tatsächlichen Gegenstand der Praxis ausrichtet, dürfte dagegen noch als zulässig angesehen werden. Auch bei gegenständlichen Grenzen ist eine geltungserhaltende Reduktion einer Wettbewerbsklausel auf ein noch zulässiges Maß nicht möglich, sodass eine Überschreitung ebenfalls zur Nichtigkeit der gesamten Klausel gemäß $§ 138$ Abs. 1 BGB führt.

\section{Abfindungsklausel}

Für den Fall, dass der Gesellschafter am Gesellschaftsvermögen beteiligt ist, muss im Gesellschaftsvertrag eine Abfindungsklausel vereinbart werden, welche die Beteiligung des Gesellschafters am ideellen Vermögen, dem sog. „good-will“ und an dem materiellen Vermögen der Gesellschaft im Falle des Ausscheidens kompensieren soll. Unter „good-will“ ist der immaterielle Wert zu verstehen, der im Fall einer ärztlichen Gemeinschaftspraxis in erster Linie aus Patientenbindungen und Verbindungen zu den sog. ärztlichen „Zuweisern“, also zu Haus- oder Fachärzten, die Patienten an die Gemeinschaftspraxis überweisen, besteht (LG Heidelberg, Urt. v. 30.09.2013, Az.: 5 O 104/13).

In diesem Zusammenhang ist zu beachten, dass eine illoyale Verwertung der Erfolge der gemeinsamen Arbeit dann vor- liegen kann, wenn der ausscheidende Gesellschafter eine Abfindung erhält, welche auch den Wert des „good-will“ umfasst und dieser sodann durch Konkurrenztätigkeit in zeitlicher, räumlicher und gegenständlicher Nähe zur Gesellschaft, insbesondere durch „Mitnahme“ von Geschäftsbeziehungen oder Patientenströmen, dennoch am gemeinsam aufgebauten „good-will“ partizipiert. Dem ausscheidenden Gesellschafter muss nicht gestattet werden, sich zugleich für das Zurücklassen der immateriellen Werte entschädigen $z u$ lassen und diese dennoch auszunutzen (BGH, Urt. v. 08.05.2000, Az.: II ZR 308/98).

\section{"Mitnahme“ des Vertragsarztsit- zes $\nabla$}

Daneben wird in Gemeinschaftspraxisverträgen häufig, neben einer Wettbewerbsklausel, das Schicksal des dem ausscheidenden Gesellschafter gehörenden vertragsärztlichen Versorgungsauftrags geregelt. Soll dieser dem Gesellschafter bei Austritt erhalten bleiben, ist gerade in Bezug auf die o.g. räumlichen Grenzen darauf zu achten, dass für den Ausscheidenden die Möglichkeit bestehen bleiben muss, sich in seinem Zulassungsbezirk vertragsärztlich niederlassen zu können.

Wird vereinbart, dass der Vertragsarztsitz bei Ausscheiden des Gesellschafters in der Gemeinschaftspraxis verbleiben soll und der Ausscheidende auf diesen zugunsten eines von den verbleibenden Gesellschaftern zu benennenden Nachfolgers verzichten muss, so ist anhand des Einzelfalls die Zulässigkeit der Regelung zu entscheiden. In die Abwägung einfließende Kriterien können insbesondere sein: die Dauer der Zugehörigkeit zur Gesellschaft, die Frage von wem der Vertragsarztsitz eingebracht wurde, die Höhe einer möglichen Einlage, die beruflichen und wirtschaftlichen Nachteile für die jeweilige Seite in Abhängigkeit davon, ob der Vertragsarztsitz in der Praxis verbleibt oder mitgenommen wird und die Höhe einer möglichen Abfindung. Der BGH hat entschieden, dass eine gesellschaftsvertragliche Regelung, die dem neu eingetretenen Vertragsarzt für den Fall, dass er freiwillig aus der Gemeinschaftspraxis ausscheidet, die Pflicht auferlegt, einen Antrag auf Ausschreibung des vakant werdenden Kassenarztsitzes zu stellen, jedenfalls dann nicht gegen $\S 138$ Abs. 1 BGB i. V.m. Art. 12 Abs. 1 GG verstößt, wenn der Ausschei- 
dende wegen der relativ kurzen Zeit seiner Mitarbeit die Gemeinschaftspraxis noch nicht entscheidend mitprägen konnte. In den vom BGH entschiedenen Fällen betrug dieser Zeitraum 1-2 Jahre (vgl. BGH, Urt. v. 22.07.2002, Az.: II ZR 265/00 und Urt. v. 22.07.2002, Az.: II ZR 90/01).

\section{Wettbewerbsverbote im Arbeits- vertrag}

Nachvertragliche Wettbewerbsklauseln finden auch in Arbeitsverträgen von angestellten Ärzten Eingang und unterliegen aufgrund der Schutzbedürftigkeit von angestellten Ärzten deutlich engeren Voraussetzungen, da ein berufliches Überund Unterordnungsverhältnis vermutet wird. Sie sind nur dann für die Parteien verbindlich, soweit berechtigte Interessen des Arbeitgebers geschützt werden. Probleme bereitet hierbei regelmäßig die Frage, wie weit die berechtigten Interessen des Arbeitgebers gehen dürfen.

Rechtsgrundlage für die Vereinbarung eines nachvertraglichen Wettbewerbsverbots in Arbeitsverträgen ist $\S 110$ GewO i.V.m. $\S \S 74$ ff. HGB, wonach das Wettbewerbsverbot schriftlich festzuhalten und eine Urkunde, zumeist durch Übergabe des Arbeitsvertrages, auszuhändigen ist. Gemäß $§ 74$ Abs. 2 HGB ist das Wettbewerbsverbot nur verbindlich, wenn für die Dauer des Verbots eine Karenzentschädigung gezahlt wird, die für jedes Jahr des Verbots mindestens die Hälfte der zuletzt bezogenen vertragsmäßigen Leistungen erreicht.

In zeitlicher Hinsicht darf die Wettbewerbsbeschränkung gemäß $\S 74$ a Abs. 1 S. 3 HGB die Dauer von 2 Jahren nicht übersteigen. In diesem Zusammenhang ist jedoch bei einer Überschreitung der Höchstgrenze eine Reduzierung auf das zulässige Maß durch das Gericht im Wege der geltungserhaltenden Reduktion möglich (BAG, Urt. v. 21.04.2010, Az.: 10 AZR $288 / 09)$. Sollte demgegenüber eine vereinbarte Karenzentschädigung fehlen, führt dies zur Nichtigkeit des Wettbewerbsverbots.

Die Bestimmung des sachlichen und räumlichen Geltungsbereichs gestaltet sich auch bei arbeitsvertraglich vereinbarten nachvertraglichen Wettbewerbsverboten schwierig. Begrenzt wird die Reichweite des nachvertraglichen Wettbewerbsverbots jedenfalls durch die ge- schäftlichen Interessen des Arbeitgebers. Es ist ferner unverbindlich, soweit es unter Berücksichtigung der gewährten Entschädigung nach Ort, Zeit oder Gegenstand eine unbillige Erschwerung des Fortkommens des Arbeitnehmers enthält (vgl. §74 a Abs. 1 S. 2 HGB).

Der Arbeitgeber kann sich auf ein unverbindliches Wettbewerbsverbot nicht berufen. Der Arbeitnehmer hat dagegen die Wahl: Er kann sich einerseits an das Wettbewerbsverbot halten und dafür eine Karenzentschädigung verlangen. Andererseits kann er den Wettbewerb weiterhin betreiben, bleibt jedoch im Gegenzug entschädigungslos.

\section{Fazit \\ $\nabla$}

Der Abschluss nachvertraglicher Wettbewerbsklauseln sollte zuvor von den jeweiligen Parteien gründlich durchdacht werden. In die Festlegung der Grenzen, insbesondere in räumlicher Hinsicht, sollte das Interesse der Gemeinschaftspraxis an der Existenzbewahrung und der Erhaltung des Patientenstammes und dem legitimen Interesse des Ausscheidenden am Aufbau einer neuen Existenz abgewogen werden. Von beiden Seiten sollten gleichermaßen zumutbare Einbußen hingenommen, aber auch ebenso gleiche Chancen, z.B. hinsichtlich der verbleibenden Möglichkeit der vertragsärztlichen Tätigkeit in dem Zulassungsbezirk des Ausscheidenden, zugebilligt werden.

In arbeitsrechtlicher Hinsicht ist dem Arbeitgeber anzuraten, vor Abschluss der kostenintensiven nachvertraglichen Wettbewerbsklausel genau zu prüfen, ob der Umfang der geschäftsinternen Kenntnisse des Arbeitnehmers die Kosten einer zu zahlenden Karenzentschädigung rechtfertigt.

RA Dr. Peter Wigge

Fachanwalt für Medizinrecht

Stefanie Broß

Rechtsanwältin

Rechtsanwälte Wigge

Scharnhorststr. 40

48151 Münster

Telefon: (0251) 53595-0

Telefax: (0251) 53595-99

E-Mail: kanzlei@ra-wigge.de

www.ra-wigge.de 